\title{
Robotic-arm assisted total hip arthroplasty
}

\author{
Matthew S. Hepinstall, Nipun Sodhi, Joseph O. Ehiorobo, Shazaan Hushmendy, Michael A. Mont \\ Department of Orthopaedic Surgery, Lenox Hill Hospital, Northwell Health, New York, NY, USA \\ Correspondence to: Michael A. Mont. System Chief of Joint Reconstruction Vice President, Strategic Initiatives Lenox Hill Hospital, Northwell \\ Health, New York, NY, USA. Email: Mmont@northwell.edu; rhondamont@aol.com. \\ Comment on: Qin J, Xu Z, Dai J, et al. New technique: practical procedure of robotic arm-assisted (MAKO) total hip arthroplasty. Ann Transl Med \\ 2018;6:364.
}

Submitted Oct 04, 2018. Accepted for publication Oct 12, 2018.

doi: $10.21037 / \mathrm{atm} .2018 .10 .37$

View this article at: http://dx.doi.org/10.21037/atm.2018.10.37

Despite the overall successful track record of total hip arthroplasty (THA), there is still the potential for complications related to component malpositioning, such as leg length discrepancy, dislocations and early implant failures (1). A robotic-arm or any other tool that can help increase surgical reliability through enhanced planning, accuracy and precision of component placement may therefore be of added value.

Qin and colleagues nicely highlight the use of a roboticarm for THA. This operative technology has been recently introduced in China, and the authors describe their early adoption. Specifically, they discuss some of the potential advantages, such as increased accuracy in cup positioning and improved patient satisfaction, as well as the operative techniques, which included further details of enhanced planning, and the role of team members. The authors also demonstrate an operative case.

Robotic-arm assisted surgery was introduced in the United States many years ago, and has been associated with a number of potential advantages (2,3). Illgen et al. (4) compared robotic-arm assisted $v s$. manual THAs and found the rate of acetabular component placement within Lewinnek safe zones to be the highest in the roboticarm assisted cohort $v s$. the manual THAs (77\% vs. $30 \%$; $\mathrm{P}<0.001)$. The group also found a $0 \%$ dislocation rate in the robotic-arm cohort, compared to a $5 \%$ in the manual THA cohort. Similarly, Elmallah et al. (5) evaluated 224 roboticarm assisted THAs and found $99 \%$ of patients to have components within the pre-designated safe zone. Kamara et al. (6) found greater improvements in the precision of component positioning with adoption of the robotic-arm compared to adoption of fluoroscopic guidance.
Furthermore, Bukowski et al. (7) compared 100 roboticarm vs. 100 manual THAs and found significantly lower estimated blood loss ( 374 vs. $423 \mathrm{~mL}, \mathrm{P}=0.035)$ in the robotic-arm cohort, as well as significantly greater mean modified Harris Hip Scores (92 vs. 86 points, $\mathrm{P}=0.002$ ), and mean UCLA activity scores (6.3 vs. 5.8 points, $\mathrm{P}=0.033)$ for robotic-arm assisted THA patients. Perets et al. (8) analyzed 181 robotic-arm assisted patients, and found these patients to have favorable short-term outcomes.

The robotic-arm has also been found to be advantageous for total knee arthroplasty (TKA). Specifically, the robotic-arm technique has been associated with enhanced component position accuracy and precision (9), ability to correct coronal deformities, even in severe cases (10), and protect surrounding soft-tissue $(11,12)$.

In our clinical practice, we have utilized the robotic-arm extensively for hip and knee replacement, and agree with the technical tips and observations of Qin and colleagues, such as the importance of proper probing technique for osteoporotic bone during bone registration. We also agree that although the robotic-arm is a useful tool, the surgeon should maintain ultimate responsibility and control as he/ she would during a manually performed THA.

Overall, as Qin and colleagues report, robotic-arm assistance for THA offers the potential for more reliable surgeries and outcomes. Current evidence demonstrates that this technology allows components to be placed more accurately and precisely at the surgeon's target. This can reduce post-operative complications, ultimately resulting in better patient function and greater patient satisfaction (7). Currently published studies report the results of surgeons who were early adopters of this new technology. We 
look forward to further studies documenting the effect of robotic-arm assisted surgery on patient-centered outcomes in the hands of a larger and more diverse group of surgeons.

\section{Acknowledgements}

None.

\section{Footnote}

Conflicts of Interest: MS Hepinstall: AAOS, Corin USA, KCI, Stryker; MA Mont: AAOS, Cymedica, DJ Orthopaedics, Johnson \& Johnson, Journal of Arthroplasty, Journal of Knee Surgery, Microport, National Institutes of Health (NIAMS \& NICHD), Ongoing Care Solutions, Orthopedics, Orthosensor, Pacira, Peerwell, Performance Dynamics Inc., Sage, Stryker: IP royalties, Surgical Technologies International, Kolon TissueGene. The other authors have no conflicts of interest to declare.

\section{References}

1. Delaunay C, Hamadouche M, Girard J, et al. What are the causes for failures of primary hip arthroplasties in France? Clin Orthop Relat Res 2013;471:3863-9.

2. Redmond JM, Gupta A, Hammarstedt JE, et al. Accuracy of Component Placement in Robotic-Assisted Total Hip Arthroplasty. Orthopedics 2016;39:193-9.

3. Domb BG, Redmond JM, Louis SS, et al. Accuracy of Component Positioning in 1980 Total Hip Arthroplasties: A Comparative Analysis by Surgical Technique and Mode

Cite this article as: Hepinstall MS, Sodhi N, Ehiorobo JO, Hushmendy S, Mont MA. Robotic-arm assisted total hip arthroplasty. Ann Transl Med 2018;6(22):433. doi: 10.21037/ atm.2018.10.37 of Guidance. J Arthroplasty 2015;30:2208-18.

4. Illgen RL Nd, Bukowski BR, Abiola R, et al. RoboticAssisted Total Hip Arthroplasty: Outcomes at Minimum Two-Year Follow-Up. Surg Technol Int 2017;30:365-72.

5. Elmallah RK, Cherian JJ, Jauregui JJ, et al. Robotic-Arm Assisted Surgery in Total Hip Arthroplasty. Surg Technol Int 2015;26:283-8.

6. Kamara E, Robinson J, Bas MA, et al. Adoption of Robotic vs Fluoroscopic Guidance in Total Hip Arthroplasty: Is Acetabular Positioning Improved in the Learning Curve? J Arthroplasty 2017;32:125-30.

7. Bukowski BR, Anderson P, Khlopas A, et al. Improved Functional Outcomes with Robotic Compared with Manual Total Hip Arthroplasty. Surg Technol Int 2016;29:303-8.

8. Perets I, Walsh JP, Close MR, et al. Robot-assisted total hip arthroplasty: Clinical outcomes and complication rate. Int J Med Robot 2018;14:e1912.

9. Hampp EL, Chughtai M, Scholl LY, et al. Robotic-Arm Assisted Total Knee Arthroplasty Demonstrated Greater Accuracy and Precision to Plan Compared with Manual Techniques. J Knee Surg 2018. [Epub ahead of print].

10. Marchand RC, Sodhi N, Khlopas A, et al. Coronal Correction for Severe Deformity Using Robotic-Assisted Total Knee Arthroplasty. J Knee Surg 2018;31:2-5.

11. Sultan AA, Piuzzi N, Khlopas A, et al. Utilization of robotic-arm assisted total knee arthroplasty for soft tissue protection. Expert Rev Med Devices 2017;14:925-7.

12. Khlopas A, Chughtai M, Hampp EL, et al. Robotic-Arm Assisted Total Knee Arthroplasty Demonstrated Soft Tissue Protection. Surg Technol Int 2017;30:441-6. 